\title{
AMPLIANDO AS FUNCIONALIDADES DE UM SISTEMA GERENCIADOR DE APRENDIZAGEM PARA O CONTEXTO DA TELEMÁTICA MÓVEL
}

\author{
Samuel Frezza Pisa ${ }^{1}$ \\ Igor Soares Alves ${ }^{1}$ \\ Elias José Paulino de Melo ${ }^{1}$ \\ Márcio José Pancheri ${ }^{1}$ \\ Luiz Fernando Tavares Meirelles ${ }^{1}$ \\ ${ }^{1}$ Centro Universitário Claretiano (Claretiano) - Batatais - SP \\ pancheri@claretianas.com.br, eliasjosemelo@gmail.com)
}

(luizfernando@claretiano.edu.br, samfrezza@gmail.com,igorsoaresalves@gmail.com,

\section{Resumo}

Com o uso de tecnologias como J2ME, XHTML-MP e WCSS é possível ampliar o uso de sistemas gerenciadores de aprendizagem acessados tipicamente em computadores de mesa ou notebooks para que sejam acessados utilizando-se smartphones. Apresentamos neste trabalho o resultado da experiência que obtivemos ao ampliarmos as funcionalidades do Sistema Gerenciador de Aprendizagem do Centro Universitário Claretiano para o contexto da aprendizagem com mobilidade.

\section{Palavras-chaves}

aprendizagem com mobilidade, $J 2 M E$, ead, educação a distância

\begin{abstract}
With the use of tecnologies like J2ME, XHTML-MP and WCSS it's possible to broaden the use of apprenticeship managing systems typically acessed in personal computers or notebooks so that they can be acessed in smartphones. Is presented in this work the result of the experience we obtained when we enlarged the operation of the Apprenticeship Managing System of Claretian Universitary Center for the context of mobile learning
\end{abstract}

Keywords 
mobile learning, J2ME, ead, distance education

\section{1 - Introdução}

Educadores e estudantes necessitam acompanhar os avanços tecnológicos, integrando no cotidiano do ensinar e do aprender novos recursos e serviços telemáticos. Atualmente podemos contar com dispositivos móveis e portáteis que nos permitem o acesso à rede mundial de computadores mesmo quando estamos em movimento. $\mathrm{O}$ uso de desses dispositivos está se popularizando, tornando imprescindível que atividades educacionais adotem essa tecnologia, contribuindo para a quebra do paradigma do ensino-aprendizagem em sala de aula virtual somente com o uso de computadores de mesa ou de notebooks. Ampliar as funcionalidades dos sistemas gerenciadores de aprendizagem para o contexto da telemática móvel irá permitir que o aprendizado ocorra independente de lugares e horários, ampliando as possibilidades de interação entre alunos e professores, e destes com os conteúdos.

\section{2 - Sistema Gerenciador de Aprendizagem}

Profissionais que atuam na Coordenadoria de Tecnologias da Informação e da Comunicação da Ação Educacional Claretiana desenvolveram o Sistema Gerenciador de Aprendizagem (SGA) com o propósito de apoiar atividades de ensino-aprendizagem na modalidade a distância. Após efetuar login, alunos e professores podem utilizar 10 funcionalidades gerais: "Início", "Podcast", "Boletim", "Agenda", "Meus Dados", "Biblioteca", "Webmail", "Secretaria", "Fale Conosco" e "Sair".

O SGA possui ferramentas que compõem um ambiente virtual online de aprendizagem denominado Sala de Aula Virtual (SAV). Em cada SAV podem ser habilitadas até 11 ferramentas, dependendo da necessidade de cada disciplina. As ferramentas são: "Orientações", "Material de Apoio", "Portfólio", "Fórum”, "Lista", "Bate-Papo", "Faq", "Vídeoaula”, "Referências", "Mural” e “Turma”. As ferramentas "Portfólio", "Fórum" e "Lista", por apoiarem interação assíncrona, possibilitam aos alunos e professores maior flexibilidade para o desenvolvimento de atividades de ensino-aprendizagem em ambiente online. 
$\mathrm{O}$ acesso a essas ferramentas num contexto de aprendizagem com mobilidade poderá contribuir para que as interações entre alunos e professores ocorram de forma mais frequente (ganho quantitativo) e contextualizada/significativa (ganho qualitativo).

\section{3 - Desenvolvimento de Aplicativos para Dispositivos Telemáticos Móveis e Portáteis}

Os Dispositivos Telemáticos Móveis e Portáteis (DTMP) podem ser definidos segundo Meirelles (2007), como um tipo de telefone celular, com capacidade computacional semelhante à de computadores de mão (Personal Digital Assistents $P D A$ 's), acrescido de tecnologias pertencentes a área de telefonia móvel.

Um smartphone é um DTMP voltado para o meio corporativo e pessoal, com funcionalidades que superam os celulares comuns. Um dos principais recursos que se destaca nesse dispositivo é o acesso à Internet, realizado por meio de tecnologias como GPRS, EDGE, $3 G$ e Wi-Fi.

$\mathrm{Na}$ pesquisa e nos experimentos que realizamos, adotamos o smartphone fabricado pela Nokia, modelo E62. As seguintes características estão sendo relevantes no processo de portabilidade do SGA para o suporte a aprendizagem com mobilidade:

- 2.8 polegadas (diagonal) QVGA (320 x 240 pixels), 16 milhões de cores

- teclas de silicone com superfície rígida

- botão de navegação com 5 direções, tecla liga/desliga utilizada como tecla de perfil, 2 soft keys

- teclado completo estendido (QUERTY)

- memória interna com até $80 \mathrm{Mb}$, não-volátil (memória exata depende de configurações da operadora, região e mercado)

- espaço para cartão Mini-SD (hot swap) - suporte para cartão com capacidade de até

\section{Gigabytes}

- leitor de cartão SIM com suporte para a tecnologia GSM

- conectividade EGSM 850/900/1800/1900 GPRS/EGPRS (Classe B, MSC 10) GSM CSD de até 14.4 e 43.2 (HSCSD) kbps

Nos últimos anos pode-se perceber que o uso dos DTMP vem crescendo de forma cada vez mais rápida e a evolução desses dispositivos em termos de recursos também acontece na mesma velocidade. Com o desenvolvimento tecnológico nessa área, esses dispositivos ganharam funcionalidades típicas de computadores como, por exemplo, players de imagem, áudio e vído, jogos e acesso à Internet. 
Mesmo com essa crescente agregação de novas aplicabilidades os dispositivos móveis ainda sofrem limitações em termos de memória, processamento e resolução de tela, fazendo com que softwares sejam adaptados ou desenvolvidos especificamente para o uso nesses dispositivos.

Dentre as várias linguagens e plataformas disponíveis para o desenvolvimento, identificamos no Java 2 Micro Edition $(J 2 M E)$ e na formatação de páginas web, soluções tecnológicas para os propósitos de nosso projeto, cujos resultados da análise técnica que realizamos estão descritos nas seções 3.1 e 3.2 .

\subsection{J2ME}

O J2ME é uma versão da plataforma Java para dispositivos móveis, destinada a equipamentos com poder limitado (Muchow 2004). Em geral os dispositivos móveis sofrem limitações em termos de recursos computacionais, tornando o $J 2 M E$ padrão uma plataforma com poucos recursos. Outra dificuldade relaciona-se com a capacidade de manter a portabilidade entre DTMP que se diferenciam em relação ao hardware e ao sistema operacional, pois para ser executado no dispositivo, o $J 2 M E$ necessita de uma máquina virtual Java, chamada de KVM, que é composta de várias bibliotecas denominadas JSRs, geralmente, instalada pelo fabricante. Cada fabricante compõe a máquina virtual de acordo com o sistema operacional e a capacidade do dispositivo, podendo não incluir algumas bibliotecas opcionais, impossibilitando a portabilidade. Experiências com a manipulação de informações demonstrou que a recuperação e o armazenamento de dados, utilizando apenas os recursos do $J 2 M E$, poderá ser, conforme o volume, computacionalmente ineficiente.

\subsection{Projeto para Web Móvel}

O projeto para web móvel (Mobile Web Design) permite que o desenvolvedor crie páginas web que serão lidas com um certo grau de padronização pelos diversos navegadores em diferentes DTMP. Diante das limitações de tais aparelhos o projeto para web móvel define características que possibilitam ao usuário uma navegação rápida e confortável.

O XHTML Mobile Profile (XHTML-MP) foi criado pela Open Mobile Aliance (OMA) com base no XHTML-Basic. Voltado para o mundo mobile, o XHTML-MP usa a 
mesma sintaxe do XHTML, utilizado em sites convencionais. Com apenas algumas adaptações facilita o trabalho do desenvolvedor, que não precisará aprender uma nova linguagem, além de ser interpretada por qualquer navegador web.

Com o auxilio do WAP Cascading Style Sheet (WCSS) o layout do site na versão mobile pode ser controlado facilmente e com a segurança de que será lido por todos os navegadores que seguirem os padrões da OMA. O WCSS é uma versão simplificada do CSS, folhas de estilo utilizadas para separar o formato das páginas a serem exibidas de seus conteúdos, facilitando alterações.

\section{4 - Portando o SGA-SAV para DTMP}

A primeira tecnologia que utilizamos para portar o SGA-SAV (Figura 1) para dispositivos telemáticos móveis e portáteis foi $J 2 M E$. Entretanto, devido às suas limitações quanto a processamento, armazenamento de dados e recursos gráficos, optou-se por tecnologias destinadas ao desenvolvimento de projeto para web móvel.

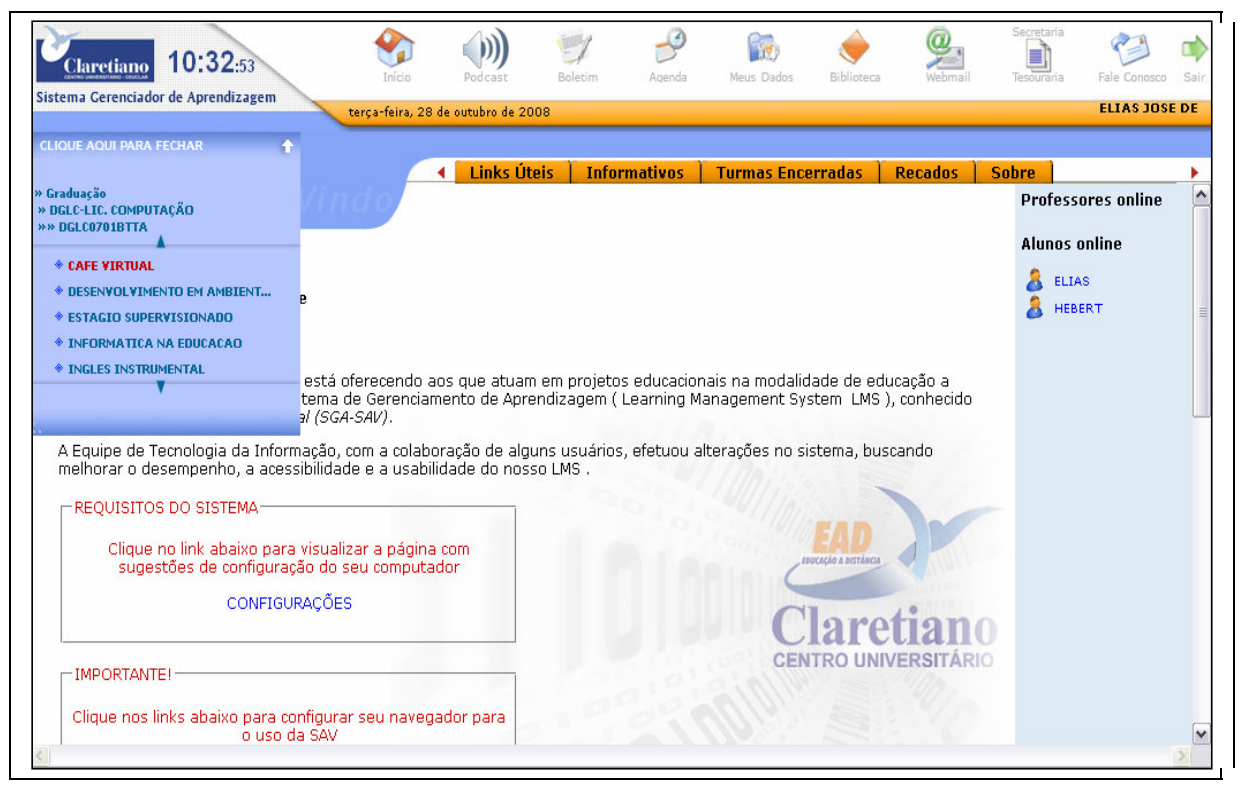

Figura 1 - Página inicial do SGA-SAV

Cabe observar que o desenvolvimento estava focado em smartphones e que, o dispositivo E62 da Nokia foi adotado com o intuito de que a versão móvel do SGASAV, denominada mSAV, pudesse ser acessada, tanto por smartphones que apresentam 
todos os recursos tecnológicos disponíveis no mercado, quanto por aqueles mais simples que só possuem recursos padrões como, por exemplo, o acesso à Internet, o que torna o desenvolvimento para web uma alternativa viável para os objetivos do projeto.

Para desenvolver a mSAV (Figuras 2 e 3) o primeiro procedimento foi elaborar a parte gráfica, com um layout que deveria ter uma estrutura focada nas características dos smartphones. Durante o desenvolvimento foram consideradas algumas questões como o tamanho da tela, recursos disponíveis, navegabilidade e quantidade de dados contido em cada página, caracterizando um cenário diferente do que temos quando o desenvolvimento está direcionado para computadores de mesa ou notebooks.

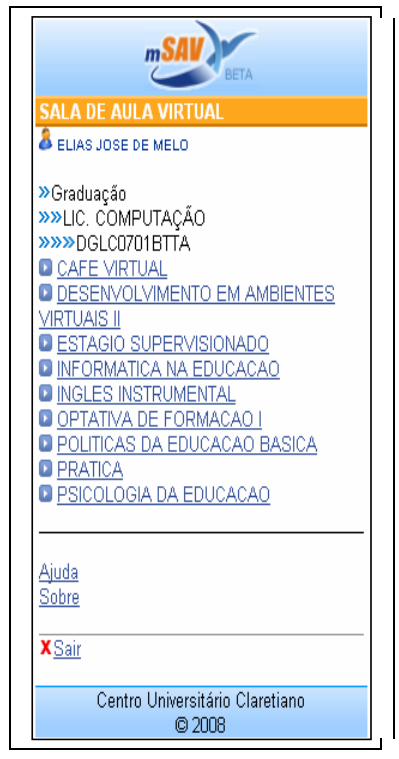

Figura 2 - Página Inicial mSAV

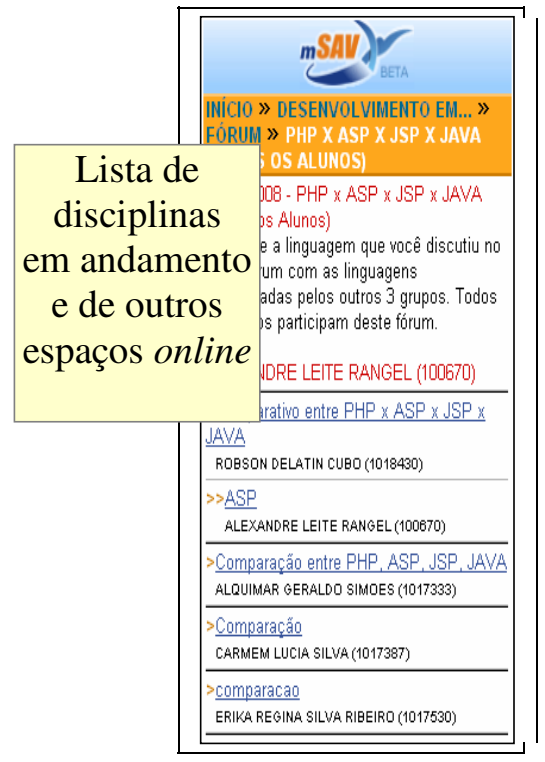

Mensagem
de abertura
e árvore de
mensagens
no Fórum

Figura 3 - Fórum mSAV

Concluída a estrutura do layout o próximo passo foi escolher as ferramentas e funcionalidades do SGA-SAV que seriam portadas. Utilizamos como critérios a viabilidade técnica e a utilidade pedagógica. Identificando o que seria viável tecnicamente e útil pedagogicamente, foram escolhidas as ferramentas de apoio a interação: o "Portfólio", a "Lista" e o "Fórum". Na portabilidade dessas ferramentas foi necessário adotar as seguintes premissas de projeto:

- Utilização de imagens com tamanho reduzido, visando diminuir o tráfego de dados e tornar a navegação mais rápida.

- Adaptação da navegação, na qual o rolamento horizontal foi evitado, a fim de tornar o aplicativo mais funcional. 
- Não utilização da linguagem Javascript, com objetivo de poupar recursos de processamento e devido à uma falta de padronização entre os navegadores disponíveis nos DTMP.

- Criação de menu hierárquico para facilitar a navegação do usuário.

Terminada a estruturação dos componentes de interface para o aplicativo foram iniciados estudos para a implementação de um mecanismo computacional que permitisse a transferência de dados entre o SGA-SAV e o mSAV. Optamos pelo desenvolvimento de um "Mediador mSAV", responsável pelo fornecimento de informações através de serviços, conforme o diagrama apresentado na Figura 4.

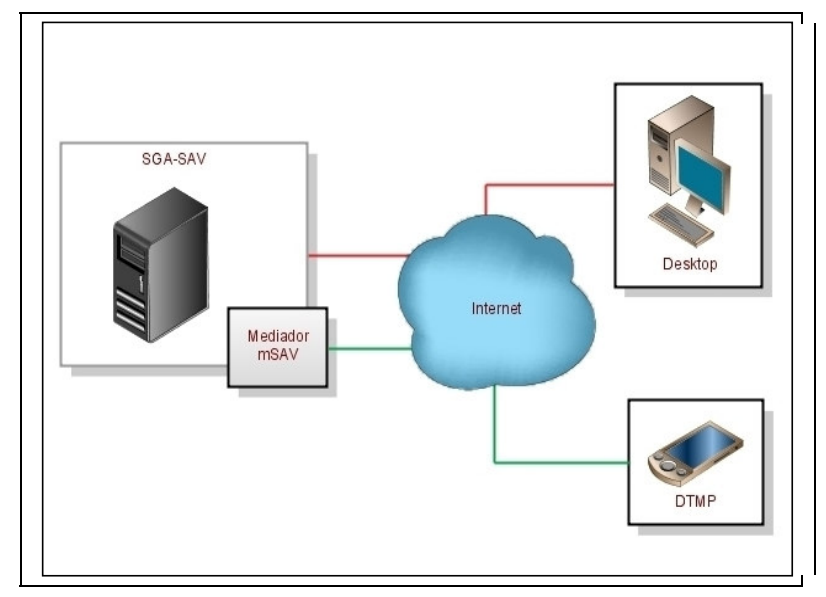

Figura 4 - Diagrama simplificado dos componentes do mSAV.

O Mediador mSAV é um módulo que faz parte do SGA-SAV e foi adotado para manter a integridade dos dados e evitar alterações na estrutura do sistema, disponibilizando um novo tipo de acesso as informações da base de dados.

Em seu funcionamento o navegador mobile envia parâmetros ao serviço com a ação desejada e os dados necessários para a sua realização como, por exemplo, identificação do aluno e senha. Com esses parâmetros o Mediador mSAV envia uma requisição ao servidor e retorna as informações no DTMP do usuário.

Os testes que realizamos, embora restritos ao grupo que atua no projeto e em condições telemáticas até certo ponto controladas, foram plenamente satisfatórios quanto a usabilidade e o desempenho, fatores que consideramos relevantes para que soluções tecnológicas tipo mSAV sejam efetivamente adotadas. 


\section{5 - Conclusão e Trabalhos Futuros}

Os estudos e experimentos que realizamos, permitiram identificar nas tecnologias relacionadas ao projeto para web móvel uma alternativa mais eficiente para o desenvolvimento de versões mobile de sistemas gerenciadores de aprendizagem. Com a tecnologia disponível atualmente o acesso ao SGA-SAV, na versão móvel denominada mSAV é perfeitamente viável, permitindo que alunos e professores participem das discussões com maior flexibilidade de tempo e espaço. Com o surgimento de novas tecnologias como a banda larga móvel será possível portar outras ferramentas do SGA-SAV como, por exemplo, "Podcast" e "Vídeoaulas". Para o próximo período letivo (ano de 2009) a versão mobile do SGA-SAV (mSAV) será disponibilizada aos alunos e professores, possibilitando validar e aprimorar a portabilidade do sistema num cenário de uso real. Fatores tecnológicos relacionados a conectividade, a usabilidade e a compatibilidade com diferentes DTMP, juntamente com possibilidades, implicações e inovações didático-pedagógicas, são temas futuros de nossa investigação.

\section{6 - Bibliografia e Referencias Bibliográficas}

Cremin, Ronan et al. Mobile Web Developer's Guide - Part I:Creating a Simple Mobile Sites For Common Handsets. Disponível em: $<$ http://dev.mobi/content/dotmobi-mobile-web-developers-guide>. Acessado em 4 de agosto de 2008.

Meirelles, Luiz Fernando T. APRENDIZAGEM COM MOBILIDADE: aplicabilidade de smartphones na educação a distância. Proposta de Tese de Doutorado - PPGIE / UFRGS, 2007.

Muchow, John W. Core J2ME - Tecnologia \& MIDP - São Paulo: Pearson Makron Books, 2004.

Suda, Brian e Cors, Chuck. Designing and Developing mobile web sites in the real world, part 1. Disponível em <http://dev.opera.com/articles/view/designing-anddeveloping-mobile-web-site/>. Acessado em 4 de agosto de 2008. 